\title{
Fatores de risco à senilidade na transição à aposentadoria
}

Senility risk factors upon retirement transition

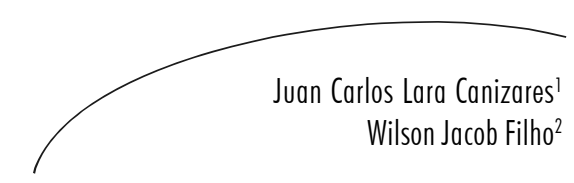

Resumo

Introdução: A aposentadoria, um dos principais desencadeantes de comprometimentos funcionais em quem envelhece, passa muitas vezes despercebida quanto a sua importância patogênica e, portanto, não recebe a devida prevenção e tratamento. Neste estudo levantou-se a hipótese de que a aposentadoria é um processo gradativo de perdas que se relaciona com o envelhecimento patológico. Objetivo: Detectar fatores psicológicos e sociais da aposentadoria determinantes no envelhecimento patológico. Casuística e Métodos: Durante o ano de 2008, 79 funcionários do Hospital das Clínicas de São Paulo, em transição à aposentadoria, foram convidados a preencher um questionário geral de opinião, de múltiplas opções de respostas, sobre fatores psicossociais da aposentadoria que melhor expressam os sentimentos e emoções perante a proximidade desse evento. Resultados: Da população de estudo, a aposentadoria afeta mais a estrutura psicológica dos homens, dos mais jovens, dos que têm menor nível de autoridade no cargo e menor nível de escolaridade. As dificuldades decorrentes do afastamento do trabalho são maiores para sujeitos mais velhos, para as pessoas com menor nível de autoridade e para pessoas com menor nível de escolaridade. A mudança de ritmo de vida decorrente da aposentadoria afeta mais sujeitos com menor nível de autoridade, e a possibilidade de surgirem conflitos após a aposentadoria é maior em sujeitos com maior nível de escolaridade. Conclusão: A aposentadoria é um processo gradativo de perdas que produz instabilidade emocional, com consequências nocivas ao futuro. Quanto menor for a idade do sujeito que se aposenta, maior o impacto desse evento. Sujeitos com maior escolaridade e nível de autoridade têm melhor adaptação à mudança e capacidade de lidar com as dificuldades na transição à aposentadoria.

\footnotetext{
1 Faculdade de Medicina da Universidade de São Paulo (LIM 01). São Paulo, SP, Brasil.

2 Faculdade de Medicina da Universidade de São Paulo, Departamento de Patologia. São Paulo, SP, Brasil.
}

Palavras-chave:

Aposentadoria. Fatores de risco. Senilidade premature.Trabalho. 
Abstract

Introduction: Retirement, one of the main drivers of functional disorders to the elderly is sometimes overlooked as regards its pathogenic significance, and, accordingly, is not provided with due care and prevention. This study approached the assumption that retirement is a pathological aging-related gradual losing process. Purpose: To identify the retirement social and psychological factors determining the pathological aging. Case Study and Methods: During 2008, 79 retiredto-be employees of the University Hospital were invited to answer a general multiple choice opinion questionnaire in connection with the retirement social and psychological factors that better express the feelings and emotions before the proximity of such event. Results: Based on the population under study, retirement mostly affects the psychological structure of men, younger individuals, people with lesser authority level in their positions and lower education level. The hindrances arising from absence from work are greater for the elderly, those with lesser authority level and those with lower education level. Life style change as a result of retirement mostly affects those individuals with lesser authority level, with a greater occurrence of post-retirement conflicts with higher education people. Conclusion: Retirement is a gradual losing process causing emotional instability with bad consequences for the future. The younger the retiring individual, the greater the retirement impact. People with higher education and authority levels show a better adjustment to such changes and a greater ability to handle any difficulties during the retirement transition.
Key words: Cost of Illness. Aged. Health of the Elderly. Retirement. Risk Factors. Aging. Premature. Job.

\section{INTRODUÇÃO}

O trabalho ocupa inegável espaço na existência humana, constituindo o principal regulador $\mathrm{da}$ organização da vida. ${ }^{1,2}$ A perda desse vínculo, além de ser fator de desequilíbrio emocional e social, ${ }^{3,4}$ pode ser determinante do comprometimento funcional, ${ }^{5,6}$ manifestando-se a partir da adoção de atitudes sedentárias, da dependência química ${ }^{5}$ e dos hábitos alimentares inadequados. ${ }^{7}$

As principais linhas de abordagens de pesquisas de pós-graduação, nas últimas três décadas, na Universidade de São Paulo, ${ }^{8}$ indicam que dentre as causas mais frequentes que levam ao afastamento precoce do trabalho destacam-se hipertensão e doenças cardiovasculares, ${ }^{9,10}$ transtornos mentais e violência, ${ }^{9,11}$ doenças osteoarticulares, infectocontagiosas, ${ }^{9,12}$ invalidez produto de traumas e quedas, ${ }^{11}$ diabetes ${ }^{11,13,14}$ estresse e sofrimento. ${ }^{15,16}$ Evidências de pesquisas, no âmbito internacional, apontam, dentre as principais conclusões, que doenças presentes nos anos que antecedem à aposentadoria podem acentuar-se após esse evento se as condições de vida forem adversas. ${ }^{4,17,18}$ Dentre as decorrências mais citadas encontram-se a dependência química, ${ }^{3}$ a depressão, ${ }^{19,20}$ as doenças cardiorespiratórias, ${ }^{21} \mathrm{a}$ obesidade $^{7} \mathrm{e}$ o estresse. ${ }^{22}$

O processo de envelhecimento e a aposentadoria ocorrem de maneiras diversas, apresentando múltiplas interfaces entre eles, relacionadas às mudanças na vida social e no mundo do trabalho. ${ }^{23} \mathrm{Da}$ mesma forma que o processo de envelhecimento, os mecanismos de enfrentamento às mudanças com ocasião da aposentadoria diferem de um sujeito para outro.

Efeitos fisiopatológicos da aposentadoria na senilidade acontecem quando esse evento se produz em condições desfavoráveis atribuídas ao estilo de vida inadequado. Portanto, torna-se necessário identificar barreiras de adaptação a uma nova fase da vida, as que possam dificultar que as pessoas tenham maiores oportunidades de permanecerem ativas e saudáveis após aposentadas. Assim, este estudo objetivou detectar fatores psicológicos e sociais da aposentadoria determinantes no envelhecimento patológico. 
Foram abordados fatores de risco determinantes da senilidade ou envelhecimento patológico presentes na transição à aposentadoria, entendendo por senilidade o conjunto de alterações decorrentes de doenças e hábitos de vida que acompanham o indivíduo durante o seu processo de envelhecimento. ${ }^{24}$

\section{CASUÍSTICA E MÉTODOS}

Foram convidados a participar da pesquisa funcionários do Instituto Central do Hospital das Clínicas de São Paulo, com idade igual ou superior a 48 anos, no caso das mulheres, e igual ou superior a 53 anos, no caso dos homens, de acordo com o critério exigido pela Previdência Social para a concessão do benefício $\mathrm{da}$ aposentadoria, de acordo com a Lei $n^{\circ} 10.666$, de 08/04/2003, publicada no registro oficial da União.

Nesse critério de corte foram identificados 1.362 funcionários, o que corresponde a 24,13\% de uma população de 5.643 servidores que trabalham no Instituto Central. Desse universo da população, 79 funcionários $(5,80 \%)$ participaram voluntariamente, durante o período de 2008 , do programa de preparação para a aposentadoria, uma atividade institucional que objetivou a orientação e o aconselhamento sobre a aposentadoria, independentemente do tempo que faltava para ela acontecer.

O estudo foi descrito como uma pesquisa transversal, aplicada, de abordagem quantitativa, que visou à análise da correlação das variáveis gênero, idade, nível de escolaridade e nível de autoridade no exercício do trabalho com fatores psicológicos e sociais da aposentadoria. Quanto aos procedimentos de coleta e de análise dos dados, foi elaborado um questionário geral de opinião de múltiplas opções de respostas, a partir das referências bibliográficas sobre fatores psicossociais da aposentadoria que melhor expressam os sentimentos e emoções perante a proximidade desse evento, foi construída uma planilha de dados no programa computacional Excel, e o tratamento estatístico das informações feito no software aplicativo SPSS (Statistical Packeage for the Social Sciences) para a descrição da relação de variáveis do estudo (correlação de Spearman).

\section{RESULTADOS}

As correlações entre as variáveis foram agrupadas em fatores psicológicos, em que se buscou conhecer a opinião das pessoas sobre aspectos emocionais associados à transição à aposentadoria (tabela 1 e 2); fatores sociais, buscando conhecer a opinião sobre o significado que a aposentadoria tem para as pessoas e os principais motivos por que esse evento passa a apresentar dificuldades de adaptação (tabela 3); e fatores associados ao estilo de vida, para conhecer a opinião sobre as possíveis mudanças nos hábitos de vida e seu impacto na saúde.

Os resultados sobre fatores de risco psicológicos descritos na tabela 1 mostram que a aposentadoria afeta mais os homens, as pessoas mais novas, com menor nível de autoridade no cargo e as pessoas com menor nível de escolaridade. Ainda dentro dos fatores psicológicos, a tabela 2 mostra que na transição à aposentadoria, as pessoas que mais manifestaram sentir ansiedade / estresse correspondem ao grupo de sujeitos com menor escolaridade e menor nível de autoridade. 
Tabela 1- Fatores de riscos psicológicos associados à estrutura emocional do sujeito na transição à aposentadoria. ICHC. São Paulo, SP, 2008.

\begin{tabular}{|c|c|c|c|c|}
\hline Variável & $\mathrm{n}$ & $\begin{array}{l}\text { Coeficiente de } \\
\text { correlação }\end{array}$ & $\begin{array}{l}\text { Significância } \\
\text { (p) }\end{array}$ & Observação \\
\hline Gênero & 77 & 0,283 & 0,013 & $\begin{array}{l}\text { Quando se pensa em aposentadoria, o sentimento } \\
\text { de tristeza afeta mais os homens. }\end{array}$ \\
\hline Idade & 61 & $-0,269$ & 0,036 & $\begin{array}{l}\text { Quanto maior a idade, menor a rejeição em } \\
\text { pensar sobre a aposentadoria. }\end{array}$ \\
\hline Idade & 61 & 0,345 & 0,007 & $\begin{array}{l}\text { Quanto maior a idade, maior o interesse sobre a } \\
\text { aposentadoria. }\end{array}$ \\
\hline Autoridade & 74 & $-0,338$ & 0,003 & $\begin{array}{l}\text { Quanto maior o nível de autoridade, menor o } \\
\text { sentimento de vazio pelo afastamento do } \\
\text { trabalho. } \\
\text { Ouanto maior o nível de autoridade menor o }\end{array}$ \\
\hline Autoridade & 77 & $-0,235$ & 0,040 & sentimento de preocupação com a aposentadoria. \\
\hline Autoridade & 77 & $-0,313$ & 0,006 & $\begin{array}{l}\text { Quanto maior o nivel de autoridade, m enor o } \\
\text { sentimento de medo com a aposentadoria. }\end{array}$ \\
\hline Escolaridade & 77 & $-0,230$ & 0,044 & $\begin{array}{l}\text { Quanto maior a escolaridade, menor o sentimento } \\
\text { de preocupação com a aposentadoria. }\end{array}$ \\
\hline
\end{tabular}

Tabela 2 - Fatores de riscos psicológicos associados à ansiedade e ao estresse presentes na transição à aposentadoria. ICHC. São Paulo, SP, 2008.

\begin{tabular}{ccccc}
\hline Variável & $\mathrm{n}$ & $\begin{array}{c}\text { Coeficiente de } \\
\text { correlação }\end{array}$ & $\begin{array}{c}\text { Significância } \\
(\mathrm{p})\end{array}$ & \multicolumn{1}{c}{ Observação } \\
\hline Escolaridade & 76 & $-0,241$ & 0,036 & $\begin{array}{l}\text { Quanto maior a escolaridade, menor a incerteza } \\
\text { quanto ao que fazer ao aposentar-se. } \\
\text { Quanto maior a escolaridade, menor a ansiedade } \\
\text { produzida pela aposentadoria. }\end{array}$ \\
Escolaridade & 63 & $-0,274$ & 0,030 & $\begin{array}{l}\text { Quanto maior o nível de autoridade, menor a } \\
\text { ansiedade produzida pela aposentadoria. }\end{array}$ \\
\hline Autoridade & 63 & $-0,318$ & 0,011 &
\end{tabular}

Os resultados sobre fatores de risco sociais na transição à aposentadoria, descritos na tabela 3 , mostram que as dificuldades decorrentes do afastamento do trabalho são maiores para sujeitos mais velhos, para as pessoas com menor nível de autoridade e para pessoas com menor nível de escolaridade. Ainda dentro dos fatores sociais, a tabela 4 mostra que formas de isolamento e exclusão social, decorrentes da aposentadoria, afetam mais os sujeitos mais velhos e os com menor nível de autoridade, assim como de escolaridade. 
Tabela 3 - Fatores de riscos sociais associados ao afastamento do trabalho na transição à aposentadoria. São Paulo, SP, 2008.

\begin{tabular}{|c|c|c|c|c|}
\hline Variável & $\mathrm{n}$ & $\begin{array}{l}\text { Coeficiente de } \\
\text { correlação }\end{array}$ & $\begin{array}{l}\text { Significância } \\
(\mathrm{p})\end{array}$ & Observação \\
\hline Idade & 73 & 0,251 & 0,032 & $\begin{array}{l}\text { Quanto maior a idade, maior a dificuldade de } \\
\text { aceitação do afastamento do trabalho. } \\
\text { Quanto maior o nível de autoridade, menor a }\end{array}$ \\
\hline Autori dade & 76 & $-0,313$ & 0,006 & $\begin{array}{l}\text { identificação da diminuição da renda como causa do } \\
\text { estresse. }\end{array}$ \\
\hline Escolaridade & 79 & $-0,231$ & 0,041 & $\begin{array}{l}\text { Quanto maior a escolaridade, menos se relaciona a } \\
\text { aposentadoria com o desvincular-se do trabalho. }\end{array}$ \\
\hline Escolaridade & 79 & $-0,314$ & 0,005 & $\begin{array}{l}\text { Quanto maior a escolaridade, menos se relaciona a } \\
\text { aposentadoria com a diminuição da renda. }\end{array}$ \\
\hline Escolaridade & 75 & $-0,513$ & $<0,001$ & $\begin{array}{l}\text { Quanto maior a escolaridade, menor a preocupação } \\
\text { com a diminuição da renda. }\end{array}$ \\
\hline & & & & $\begin{array}{l}\text { Quanto maior a escolaridade, menor as } \\
\text { preocupações com dificuldades financeiras }\end{array}$ \\
\hline Escolaridade & 74 & $-0,356$ & 0,002 & $\begin{array}{l}\text { relacionadas ao afastamento do trabalho. } \\
\text { Quanto maior a escolaridade, menos se relaciona a }\end{array}$ \\
\hline Escolaridade & 76 & $-0,249$ & 0,030 & estresse. \\
\hline
\end{tabular}

Tabela 4 - Fatores de riscos sociais associados às formas de isolamento e exclusão social produto da aposentadoria. São Paulo, SP, 2008.

\begin{tabular}{|c|c|c|c|c|}
\hline Variável & $\mathrm{n}$ & $\begin{array}{l}\text { Coeficiente de } \\
\text { correlação }\end{array}$ & $\begin{array}{l}\text { Significância } \\
\text { (p) }\end{array}$ & Observação \\
\hline Idade & 71 & 0,280 & 0,018 & $\begin{array}{l}\text { Quanto maior a idade, mai or a dificuldade de } \\
\text { retorno ao mercado de trabalho. }\end{array}$ \\
\hline Autoridade & 76 & $-0,248$ & 0,031 & $\begin{array}{l}\text { Quanto maior o nível de autoridade, menos se } \\
\text { relaciona a aposentadoria com a exclusão social. } \\
\text { Quanto maior o nível de autoridade, menor a }\end{array}$ \\
\hline Autoridade & 71 & $-0,358$ & 0,002 & $\begin{array}{l}\text { consideração de perda de status com a } \\
\text { aposentadoria. }\end{array}$ \\
\hline Escolaridade & 71 & $-0,374$ & 0,001 & $\begin{array}{l}\text { Quanto maior a escolaridade, menos considera -se a } \\
\text { perda do status profissional como forma de } \\
\text { exclusão social. }\end{array}$ \\
\hline Escolaridade & 76 & $-0,257$ & 0,025 & $\begin{array}{l}\text { Quanto maior a escolaridade, menos se relac iona a } \\
\text { aposentadoria com a exclusão social. }\end{array}$ \\
\hline
\end{tabular}

Os resultados sobre fatores de risco associados ao estilo de vida, descritos na tabela 5 , mostram que as mudanças do ritmo de vida decorrentes da aposentadoria afetam mais os sujeitos com menor nível de autoridade, e a possibilidade de surgirem conflitos após a aposentadoria é maior em sujeitos com maior nível de escolaridade. 
Tabela 5 - Fatores de riscos da aposentadoria associados ao estilo de vida e às mudanças do ritmo de vida. São Paulo, SP, 2008.

\begin{tabular}{|c|c|c|c|c|}
\hline Variável & $\mathrm{n}^{\mathrm{o}}$ & $\begin{array}{l}\text { Coeficiente de } \\
\text { correlação }\end{array}$ & $\begin{array}{l}\text { Significância } \\
\text { (p) }\end{array}$ & Observação \\
\hline Autoridade & 76 & $-0,295$ & 0,010 & $\begin{array}{l}\text { Quanto maior o nível de autoridade, menor o } \\
\text { estresse causado pela administração do tempo livre. } \\
\text { Quanto maior a escolaridade, maior a preocupação } \\
\text { com surgimento de conflitos familiares, decorrentes } \\
\text { da mudança de vida após a aposentadoria. }\end{array}$ \\
\hline
\end{tabular}

\section{DISCUSSÃO}

A condição da saúde do indivíduo idoso está atrelada ao estilo de vida nos anos que antecedem à terceira idade e, por conseguinte, no período de atividade laborativa prévio à aposentadoria. ${ }^{25}$ Maus hábitos, como tabagismo, dependência química, hábitos alimentares inadequados e sedentarismo, podem ser adquiridos durante o exercício profissional e acentuar-se na aposentadoria ou adquiridos em decorrência de estados depressivos motivados pelo processo de perdas associadas ao afastamento do trabalho (condição financeira, relacionamentos, rotina, status), tornando o sujeito vulnerável a doenças decorrentes de estilo de vida pouco saudável.

Com o processo do envelhecimento, é comum que perdas e ganhos na vida do sujeito aconteçam. Com a chegada da aposentadoria, essa percepção fica mais evidente pelo fato de se tratar de uma situação de mudança em que perdas e ganhos acontecem. Atrelar a aposentadoria à diminuição da renda é uma prática usual em sujeitos na transição à aposentadoria. É muito comum vermos profissionais maduros, no auge da sua carreira, sentirem ansiedade quanto à proximidade a aposentadoria. Um dos principais motivos desse sentimento de apreensão é o impacto da condição financeira nas fontes de renda. ${ }^{26}$

Para uma parte da sociedade, a cessação da fonte de renda do trabalho desestabiliza a estrutura socioeconômica, afetando o padrão de vida do aposentado. ${ }^{1,26,27}$ Assim, a diminuição da renda se torna uma das principais fontes de preocupação decorrente da aposentadoria. As pessoas, ao se aposentarem, deixam de fazer aquilo que por um período de tempo representou ganhos financeiros, realização profissional e pessoal, sendo natural que a proximidade desse evento gere expectativas e, mais ainda, quando essa mudança se torna uma ameaça ao padrão de vida.

$\mathrm{Na}$ fase que antecede a aposentadoria, é natural que a transição a esse evento represente ao sujeito um momento estressante, de muita expectativa e iniba sua capacidade de adaptação a situações novas ou emergentes. Quando a aposentadoria ocorre em circunstâncias adversas, como no surgimento de dificuldades de adaptação às mudanças, medo da instabilidade econômica, isolamento, dificuldade de relacionamento com a família e amigos ou desequilíbrio da estrutura emocional, ${ }^{27,28}$ ela pode tornar-se um fator de risco determinante do envelhecimento patológico.

Em situações de adversidade, as respostas diferem de intensidade de um sujeito para outro. Neste estudo verificou-se que a aposentadoria afeta mais a estrutura emocional do gênero masculino $(p=0,013)$, tornando-o mais vulnerável a quadros de tristeza, sofrimento e desânimo, podendo originar outros problemas de ordem social (conflitos familiares) com importante impacto negativo na qualidade de vida.

Neste estudo também se constatou que a idade tem relação com a dificuldade em se aposentar, fazendo com que sujeitos com idade menor encontrem problemas em lidar com o afastamento definitivo do trabalho. Por outro lado, verificou- 
se que a dificuldade de aceitação do afastamento do trabalho aumenta conforme a idade $(p=0,032)$, tendo como um dos motivos a dificuldade de retorno ao mercado de trabalho.

Atingir altos níveis de escolaridade e de autoridade no cargo é uma condição favorável para lidar com a aposentadoria, o que faz com que o sujeito se sinta mais otimista na busca de superar desafios decorrentes da mudança de hábitos e ritmo de vida. Neste estudo foi observado, ainda, que sujeitos com níveis superiores de autoridade no trabalho, na préaposentadoria, têm melhores condições de administração do tempo livre $(p=0,010)$ e que o surgimento de conflitos decorrentes da aposentadoria é menor em sujeitos com maior nível de escolaridade $(\mathrm{p}=0,010)$. Evidenciou-se que quanto maior o nível de autoridade $(p=0,003)$, melhor a condição funcional de suporte à estrutura emocional $(p=0,006)$ e

\section{REFERÊNCIAS}

1. Andujar AM. Modelo de qualidade de vida dentro do domínio biopsicossocial para aposentados. Tese [ Doutorado em Engenharia da produção]. -Universidade Federal de Santa Catarina; 2004. 208 p.

2. Zanelli V. Influências da aposentadoria na identidade pessoal. Santa Catariana [Graduação em Psicologia].- Universidade do Sul de Santa Catarina; 2007. 58p.

3. Bacharach S, Bamberg P, Sonnensthl WJ, Vashdi DR. Retirement and drug abuse: the conditioning role of age and retirement trajetory. Additive Behaviors 2008; 33(12): 16101614.

4. Kim J, Moen P. Retirement transitions, Gender, and Psychological Well-Being: a life-Course, ecological model. J Gerontol: psychological science 2008. 57(3): 212-222.

5. Queiroz B. The determinants of male retirement in urban Brazil. Nova economia 2007 jan/apr ; 17(1): 70-3

6. Bellusci SM. Envelhecimento e condições de trabalho em servidores de uma instituição judiciária . São Paulo. Dissertação [Mestrado em saúde Pública] - Faculdade de Saúde Pública; $1998.145 \mathrm{p}$. menor o nível de ansiedade $(\mathrm{p}=0,011)$ e de preocupação $(p=0,040)$ decorrentes da mudança de condição social.

\section{CONCLUSÕES}

A aposentadoria, raramente estudada como um fator de risco à saúde, muitas vezes não recebe as devidas intervenções que poderiam minimizar os efeitos de fatores de risco presentes na transição a essa nova condição social. Ela representa um processo gradativo de perdas que se relaciona com o envelhecimento patológico e que produz instabilidade emocional e desequilíbrio do padrão de vida, com consequências nocivas ao futuro. Por outro lado, o maior nível de escolaridade e de autoridade no cargo revelou-se como uma condição favorável para lidar com as dificuldades na transição à aposentadoria e para a adaptação à mudança de condição social de vida.

7. Forman-Hoffman VL, Richardson KK, Yankey JW, Hillis SL, Wallace RB, Wolinsky FD.

Retirement and weight changes among men and women in the health and retirement study. J Gerontol 2008; 63(3): 146-153.

8. DEDALUS. Banco de dados bibliográficos da USP. São Paulo: Universidade de São Paulo. [Acesso em 24 julho 2009]. Disponível em : http://200.144.190.234/F

9. Medina MCG. Aposentadoria por invalidez no Brasil.São Paulo Dissertação [ Movimento postura e ação humana] - Faculdade de Medicina da Universidade de São Paulo; 1986. $160 \mathrm{p}$.

10. Oliveira AA. Doenças cardiovasculares: trabalho e aposentadoria por invalidez. São Paulo. Dissertação [Mestrado em Saúde Publica]-Faculdade de Saúde Pública da Universidade; 2000.

11. Biazin DT. Avaliação da capacidade funcional pós-trauma em idosos. Tese [ Doutorado em enfermagem fundamental].- Escola de Enfermagem de Ribeirão Preto. Ribeirão Preto; 2006. $155 \mathrm{p}$.

12. Filipin MDV. Orquiectomia e administração de dehidroepiandrosterona (DHEA) em ratos 
wistar infectados com Trypanosoma cruzi. Ribeirão Preto. Dissertação[ Mestrado em Biociências] - Faculdade de Ciências Farmacêuticas de Ribeirão Preto; 2007. 167 p.

13. Ruiz PML. Aposentadoria e as mudanças de vida das pessoas com diabetes tipo 2. Ribeirão Preto. Tese [Doutorado em Enfermagem na saúde do adulto] - Escola de Enfermagem de Ribeirão Preto; 2007. 147 p.

14. Tavares DMS. Condições de vida e saúde de idosos diabéticos. Tese [Doutorado em enfermagem na saúde do adulto]; 2001. 156 p.

15. Bertoncini EML. Aposentadoria precoce do trabalhador bancário: determinantes de sua ocorrência. São Paulo.Tese [Doutorado em Psicologia] _Universidade de São Paulo; 1998. $169 \mathrm{p}$.

16. Farina AS. Stress na aposentadoria: um estudo com grupos diferenciados por tipo de convívio social. Tese [ Doutorado em Psicologia]Instituto de Psicologia da Universidade de São Paulo; 1998.

17. Dona M, Wilson PP. A Systematic review of published research articles on health promotion at retirement. J Nursing Scholarship 2007; 39(:4): 330-337.

18. Heponiemi T, et al. Health, psychosocial factors and retirement intentions among finnish physicians. Occupational medicine advance access published 2008 Jun; $10(8)$ : 43-9.

19. Szinovacz M, Davey A. Retirement transitions and spouse disability: effects on depressive symptoms. J Gerontol: social science 2004. 59(6) 333-342.
20. Nimrod G. Time for old friends and grandchildren? Post-Retirement Get-Togethers and Life satisfactions. J Can Ass Leisure Studies. 2008 32(1): 21.

21. Remond WLG. Jubilación: efectos médicos y psicosociales. Acta Médica Peruana 2005; 22( 3): 12-21

22. Reitzes DC, Mutran EJ. The transition to retirement: stages and factors that influence retirement adjustment. Int $\mathrm{J}$ 2004; 59(1): 63-84.

23. Bulla L, Kaefer C. Trabalho e aposentadoria: as repercussões sociais na vida do idoso aposentado. Rev Virtual Textos e Contextos 2003 $\operatorname{dez} ; 2(2): 34-46$

24. Jacob Filho W, Fló C, Santarem J, Mónaco T. Atividade física e envelhecimento saudável. São Paulo: Atheneu; 2006.

25. Amarilho C, Carlos S. O executivoempreendedor, sua aposentadoria e o processo de afastamento do trabalho. Textos sobre envelhecimento 2005 ; 8(1) : 14-22

26. Néri MC. Renda, consumo e aposentadoria: evidências, atitudes e percepções. Ensaios Econômicos 2007; 663: 1-27.

27. Cintra TS, Ribeiro DF. O mundo do trabalho e o processo de envelhecimento: o cotidiano e as representações de aposentados. In : Anais do Anais do 3. Congresso Internacional de psicologia e 9. semana de Psicologia; 2007 set 1821. Maringa, Brasil. Maringá PR, 2007

28. Neri AL. Qualidade de vida e idade madura. 5. ed .Campinas : Papirus ; 1993. 